\title{
A critical disease approach of angiogenesis in obese patients
}

\section{Suzana Binte Hashim}

EMAN Research and Testing Laboratory, Department of Pharmacology, School of Pharmaceutical Sciences, Universiti Sains Malaysia, Penang, Malaysia

Please cite this article:

Suzana Binte Hashim. (2017). A critical disease approach of angiogenesis in obese patients, 1(1), pages 041-043.

Significance |A global problem of obesity which is strongly catalyzed via angiogenesis pathology.

\section{Graphical Abstract}
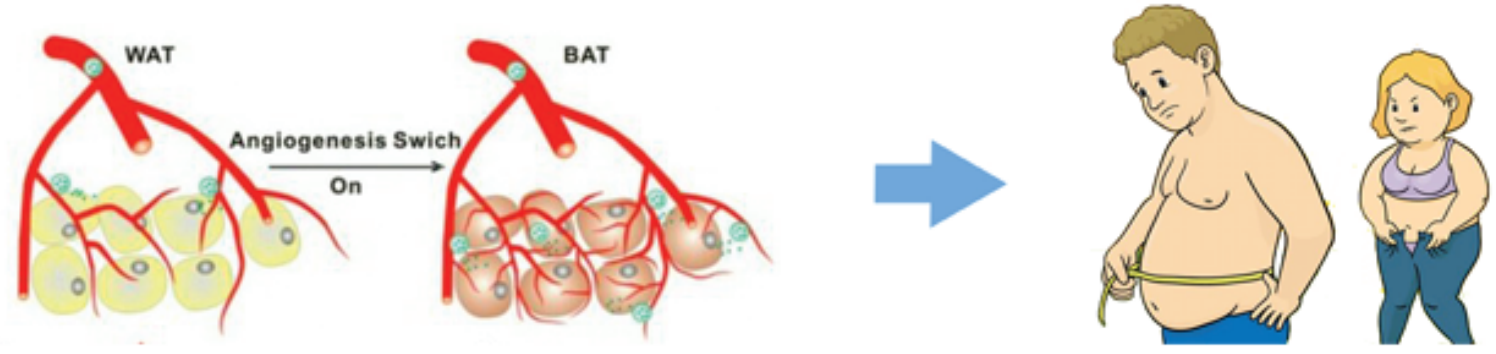

Angiogenesis switches

the obesity

${ }^{*}$ Correspondence: EMAN Research and Testing Laboratory, Department of Pharmacology, School of Pharmaceutical Sciences, Universiti Sains Malaysia, Penang, Malaysia. E-mail: hashimsuzana@yahoo.com 


\title{
A critical disease approach of angiogenesis in obese patients
}

\author{
Suzana Binte Hashim
}

\section{Abstract}

Obesity is a medical issue that is affecting an ever greater population worldwide. Also, obesity has a strong association with several metabolic disorders such as the type 2 diabetes and cardiovascular disease, which affect over $50 \%$ of an adult population (Wellen, Hotamisligil et al. 2003). Previous studies have shown that obesity facilitates the development of chronic diseases such as inflammation-based pathologies, stroke, osteoarthritis, cancer, hypertension, sleep apnoea, and arthritis (Hotamisligil 2003, Parul Singla 2010, Marta González-Castejón 2011). Importantly, the amount of cases of clinical obesity is constantly increasing, and in 2014 more than half a billion people are considered obese (WHO 2015). Therefore, it is paramount to find a solution for this problem and currently, one of the interesting avenues to combat obesity has been through angiogenesis.

Keywords: Obesity, Angiogenesis, Patients

Abbreviations: VEGF, vascular endothelial growth factor

\section{Excessive or poor angiogenesis: A balancing act}

Angiogenesis is the process by which new blood vessels form from existing blood vessel and it is a crucial natural process that occurs during healing and reproduction processes (Adair and Montani 2010). The process is controlled by the body through a

Significance |A global problem of obesity which is strongly catalyzed via angiogenesis pathology.

${ }^{*}$ Correspondence: EMAN Research and Testing Laboratory, Department of Pharmacology, School of Pharmaceutical Sciences, Universiti Sains Malaysia, Penang, Malaysia. E-mail: hashimsuzana@yahoo.com

Edited by Md Shamsuddin Sultan Khan, Hawkesbury Institute for the Environment, University of Western Sydney, Hawkesbury Campus, Bourke Street, Richmond, NSW AUSTRALIA 2753 and accepted by the Editorial Board May 8, 2017 (received for Sep 11, 2016) balanced production of pro-growth and inhibitory factors in healthy tissues. Fluctuations in this balance may lead to excessive or insufficient growth of blood vessel, which directly affects the health of an individual. Excessive blood vessel proliferation may stimulate tumor growth and spreading, rheumatoid arthritis, diabetic blindness, psoriasis, obesity and others. During excessive angiogenesis coupled with disease, new blood vessels feed the diseased tissue and can also be abnormal and "leaky", which can lead to healthy tissue damage. On the contrary, insufficient blood vessel formation causes infertility, heart disease, stroke, ulcers and scleroderma. Insufficient blood vessel formation causes improper restoration of blood circulation and increases the risk of tissue death. The link between angiogenesis and obesity is not fully established. However, the increase in proliferation and differentiation of adipocytes has been previously reported (Bråkenhielm, Cao et al. 2004, Voros, Maquoi et al. 2005, Cao 2007, Nishimura, Manabe et al. 2007, Lijnen 2008, Christiaens and Lijnen 2010) to have a strong association in the development of its vasculature. The adipose tissue undergoes expansion and regression throughout adult life and this requires parallel growth of the capillary network to support the survival of the tissues. Thus, adipose tissues are among the tissues that have highest angiogenic capacities.

\section{Adipogenesis and angiogenesis : Partners in crime VEGF influences adipogenesis}

Apart from serving as a fat storing organ, adipose tissue is important endocrine organ that secretes a variety of protein factors including chemokines, cytokines and hormone-like factors such as leptin, adiponectin and resistin (Alexander J. German a 2010). A recent review, indicated that adipogenesis is regulated by facto-

Author Affiliation:

EMAN Research and Testing Laboratory, Department of Pharmacology, School of Pharmaceutical Sciences, Universiti Sains Malaysia, Penang, Malaysia

Please cite this article:

Suzana Binte Hashim. (2017). A critical disease approach of angiogenesis in obese patients, 1(1), pages 041-043. 
rs that drive angiogenesis (Hausman and Richardson 2004). Vascular endothelial growth factor (VEGF) is the most critical growth factor involved in initiating the formation of immature blood vessels. In addition, the expression of VEGF is influenced by hypoxia, insulin, growth factors and several cytokines which are directly interconnected with the endocrine function of adipocytes. This is supported by (Nishimura, Manabe et al. 2007) in their live-cell imaging study of unfixed living adipose tissue labeled with a combination of lectin (red), BODIPY (blue), acetylated LDL (blue) and Hoechst 33342 (green) in $\mathrm{db} / \mathrm{db}$ mice. The study revealed that adipogenesis of the adipose tissues takes place within adipogenic/angiogenic cell clusters that contain various stromal cells and blood vessels. This was further validated with the administration of anti-VEGF antibodies to the mice, which showed not only an inhibition of angiogenesis but also of the formation of adipogenic/angiogenic cell clusters. This clearly indicates that coupling of adipogenesis and angiogenesis is essential for the differentiation of adipocytes in obesity and VEGF is the mediator of the process.

A review by (Daquinag, Zhang et al. 2011) on vascularization of the adipose tissue as an anti-obesity approach also indicates the roles of angiogenesis in an expansion of white adipose tissue (WAT). The development of obesity as a result of adipocyte hypertrophy (increase in cell size) as well as hyperplasia (increase in cell number) associated with the expansion of WAT which was shown to require angiogenesis. Nutrient and oxygen depletion, which inhibits WAT neovascularization, can prevent the onset of obesity in both genetic and diet-induced obesity models. This has been previously reported with the use of an anti-angiogenic drug to inhibit WAT expansion. (Rupnick, Panigrahy et al. 2002) in their study has shown that the genetically obese leptin-deficient mice from different obesity models, treated with anti-angiogenic agent (TNP-470, angiostatin (kringle 1-4 domains of plasminogen), endostatin (a C-terminal fragment of collagen XVIII), Bay-129566 (a matrix metalloproteinase inhibitor) and thalidomide) resulted in a reversible and dose-dependent weight reduction and adipose tissue loss. The treated mice showed decreased endothelial proliferation and increased apoptosis when compared with the control group, evidence that adipose tissue mass can be regulated by its vasculature. This is strongly supported by (Bråkenhielm, Cao et al. 2004) where systemic administration of the angiogenesis inhibitor TN-470 (AGM-1470) on high caloric diet-fed wt mice as well as in genetically leptin-deficient $\mathrm{ob} / \mathrm{ob}$ mice revealed a reduction in adipose tissue vascularization. This selectively affected the growth of adipose tissue, caused a decrease in insulin and serum levels of low density lipoprotein cholesterol (LDL-C), which indicates that expansion of adipose tissue can be controlled via angiogenesis inhibitor.

Another study by (Voros, Maquoi et al. 2005) on the modulation of angiogenesis during the development of adipose tissues in murine models of obesity showed that fat pad growth in both nutritionally-induced or genetically obese mice is accompanied by increased vascularization. They suggested the potential role of pro- and anti-angiogenic factors in obesity-related angiogenesis, similarly to that which was reported by (Hausman and Richardson 2004) on VEGF signaling modulation of adipose tissue development. Furthermore, (Cutchins, Harmon et al. 2012) correlated VEGF expression with the function of the inhibitor of differentiation-3 (Id3) released during the expansion of adipocytes. The expanding adipose tissue results in hypoxia, which induces VEGF expression by adipocytes. However, in the Id3-deficient mouse model, the expression of VEGFA was attenuated, leading to decreased microvascular blood volume in the adipose tissue which therefore further supports the concept that inhibition of angiogenesis in adipose tissue may prevent obesity.

\section{Natural remedies could be used to treat obesity}

Natural compounds having anti-angiogenic properties have been reported to have a positive effect on the treatment of obesity. (Kim 2010) in his pattern report, discovered that Psoraleae semen extract, Siegesbeckie herbal extract and Corni fructus extract, which exhibit anti-angiogenesis effects also demonstrated anti-obesity activity. Furthermore, (Mojzis, Varinska et al. 2008) summarized the potential function of bioactive plant flavonoids and chalcones in the modulation of angiogenesis by regulating the expression of VEGF, matrix metalloproteinases (MMPs), EGFR and inhibiting NFKB, PI3-K/Akt, ERK1/2 signaling pathways, causing strong anti-angiogenic effects. In addition, various studies on plants rich in flavonoids such as green tea extract (Chantre and Lairon 2002, Lin, Della-Fera et al. 2005), Lotus leaf extract (Du, You et al. 2010), Morus bombycis root extract (Kim, Lee et al. 2010), Nelumbo nucifera leaves extract (Ono, Hattori et al. 2006) etc. have shown significant anti-obesity effects which supports the notion that natural anti-angiogenic agents can also be promising therapeutic agents for the treatment of obesity.

\section{Conclusion}

Overall, recent studies on the modulation angiogenesis as a target by which to prevent the growth of adipose tissue may offer a better option in the treatment of obesity and other related metabolic disorder. Anti-angiogenic therapy from natural sources with promising therapeutic efficacy should be further explored to combat obesity with lower risk of side effect.

\section{Acknowledgment}

The author would like to express his gratitude to his editorial fellows.

\section{Author Contribution}

Suzana B. H. made substantial contributions to the conception of the review.

\section{Competing financial interests}

The author(s) declare no competing financial interests. 


\section{References}

Adair, T. and J. Montani (2010). Angiogenesis. Chapter 1: Overview of Angiogenesis. San Rafael (CA), Morgan \& Claypool Life Sciences.

Alexander J. German a, V. H. R., Allison C. German, I. Stuart Wood, Paul Trayhurn (2010). Obesity, its associated disorders and the role of inflammatory adipokines in companion animals. The Veterinary Journal, 185, 4-9.

Bråkenhielm, E., R. Cao, B. Gao, B. Angelin, B. Cannon, P. Parini and Y. Cao (2004). Angiogenesis Inhibitor, TNP-470, Prevents Diet-Induced and Genetic Obesity in Mice. Circulation Research, 94(12), 1579-1588.

Cao, Y. (2007). Angiogenesis modulates adipogenesis and obesity. The Journal of Clinical Investigation, 117(9), 2362-2368.

Chantre, P. and D. Lairon (2002). Recent findings of green tea extract AR25 (Exolise) and its activity for the treatment of obesity. Phytomedicine, 9(1), 3-8.

Christiaens, V. and H. R. Lijnen (2010). Angiogenesis and development of adipose tissue. Mol Cell Endocrinol, 318(1-2), 2-9.

Cutchins, A., D. B. Harmon, J. L. Kirby, A. C. Doran, S. N. Oldham, M. Skaflen, A. L. Klibanov, N. Meller, S. R. Keller, J. Garmey and C. A. McNamara (2012). "Inhibitor of differentiation-3 mediates high fat diet-induced visceral fat expansion." Arterioscler Thromb Vasc Biol, 32(2), 317-324.

Daquinag, A. C., Y. Zhang and M. G. Kolonin (2011). Vascular targeting of adipose tissue as an anti-obesity approach. Trends Pharmacol Sci, 32(5), 300-307.

Du, H., J.-S. You, X. Zhao, J.-Y. Park, S.-H. Kim and K.-J. Chang (2010). Antiobesity and hypolipidemic effects of lotus leaf hot water extract with taurine supplementation in rats fed a high fat diet. Journal of Biomedical Science, 17(Suppl 1), S42.

Hausman, G. J. and R. L. Richardson (2004). Adipose tissue angiogenesis. J Anim Sci, 82(3), 925-934.

Hotamisligil, K. E. W. a. G. S. (2003). Obesity-induced inflammatory changes in adipose tissue. J. Clin. Invest.(112), 4.

Kim, J. D. (2010). Anti-angiogenic agents and anti-obesity substances applied with anti-angiogenesis from natural products, Google Patents.

Kim, Y. S., Y. M. Lee, H. Kim, J. Kim, D. S. Jang, J. H. Kim and J. S. Kim (2010). Anti-obesity effect of Morus bombycis root extract: Anti-lipase activity and lipolytic effect. Journal of Ethnopharmacology, 130(3), 621-624.

Lijnen, H. R. (2008). Angiogenesis and obesity. Cardiovascular Research, 78(2), 286-293.

Lin, J., M. A. Della-Fera and C. A. Baile (2005). Green Tea Polyphenol Epigallocatechin Gallate Inhibits Adipogenesis and Induces Apoptosis in 3T3-L1 Adipocytes. Obesity Research, 13(6), 982-990.

Marta González-Castejón, A. R.-C. (2011). Dietary phytochemicals and their potential effects on obesity: A review. Pharmacological Research, 64, 438- 455.

Mojzis, J., L. Varinska, G. Mojzisova, I. Kostova and L. Mirossay (2008). Anti-angiogenic effects of flavonoids and chalcones. Pharmacological Research 57(4), 259-265. Nishimura, S., I. Manabe, M. Nagasaki, Y. Hosoya, H. Yamashita, H. Fujita, M.

Ohsugi, K. Tobe, T. Kadowaki, R. Nagai and S. Sugiura (2007). Adipogenesis in obesity requires close interplay between differentiating adipocytes, stromal cells, and blood vessels. Diabetes, 56(6), 1517-1526.

Ono, Y., E. Hattori, Y. Fukaya, S. Imai and Y. Ohizumi (2006). Anti-obesity effect of Nelumbo nucifera leaves extract in mice and rats. Journal of Ethnopharmacology, 106(2), 238-244.
Parul Singla, A. B., Anuj A Parkash (2010). Metabolic effects of obesity: A review. World J Diabetes, 1(3), 76-88.

Rupnick, M. A., D. Panigrahy, C. Y. Zhang, S. M. Dallabrida, B. B. Lowell, R. Langer and M. J. Folkman (2002). Adipose tissue mass can be regulated through the vasculature. Proc Natl Acad Sci U S A, 99(16), 10730-10735.

Voros, G., E. Maquoi, D. Demeulemeester, N. Clerx, D. Collen and H. R. Lijnen (2005). Modulation of Angiogenesis during Adipose Tissue Development in Murine Models of Obesity. Endocrinology, 146(10), 4545-4554.

Wellen, K. E., G. Hotamisligil, xF and S. khan (2003). Obesity-induced inflammatory changes in adipose tissue. The Journal of Clinical Investigation, 112(12), 1785-1788. WHO. (2015). Obesity and overweight.
Submit your next manuscript to Angiotherpay published by EMAN Research.

- Convenient online submission

- Thorough peer review

- No space constraints or color figure charges

- Immediate publication on acceptance

- Inclusion in Australian National Libraray and Google Scholar

- Both Open (80-100\% subsidized APC by ER) \& non-open access option

Submit your manuscript at angiotherapy.emanresearch.org 